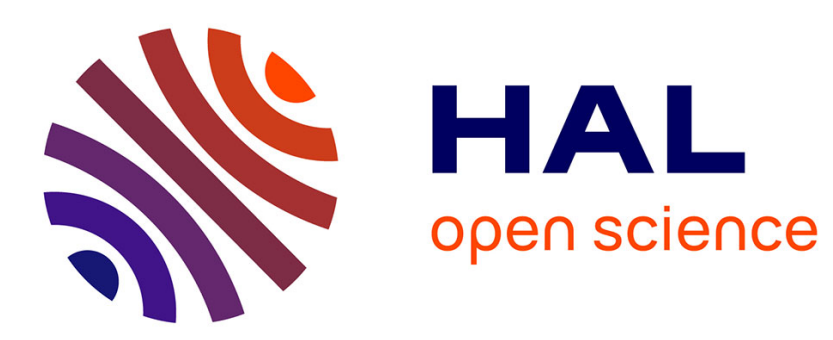

\title{
Sérialisation et hiérarchisation dans le système verbal du samba leko, langue Adamawa du Nord-Cameroun
}

\author{
Gwenaëlle Fabre
}

\section{To cite this version:}

Gwenaëlle Fabre. Sérialisation et hiérarchisation dans le système verbal du samba leko, langue Adamawa du Nord-Cameroun. Journal of African Languages and Linguistics, 2010, 30 (2), pp.231-261. 10.1515/JALL.2009.010 . hal-00937407v2

\section{HAL Id: hal-00937407 https://hal.science/hal-00937407v2}

Submitted on 29 Jan 2014

HAL is a multi-disciplinary open access archive for the deposit and dissemination of scientific research documents, whether they are published or not. The documents may come from teaching and research institutions in France or abroad, or from public or private research centers.
L'archive ouverte pluridisciplinaire HAL, est destinée au dépôt et à la diffusion de documents scientifiques de niveau recherche, publiés ou non, émanant des établissements d'enseignement et de recherche français ou étrangers, des laboratoires publics ou privés. 


\title{
SÉRIALISATION ET HIÉRARCHISATION \\ DANS LE SYSTÉME VERBAL DU SAMBA LEKO, LANGUE ADAMAWA DU NORD-CAMEROUN
}

\author{
Gwenaëlle FABRE
}

\begin{abstract}
Résumé
Le samba leko oppose deux modes marqués par deux paradigmes distincts d'indices sujet, l'assertif et l'obligatif. Chacun de ces modes dispose d'une conjugaison simple et d'une conjugaison à auxiliaire. Il découle de la simplicité d'un tel système que l'expression de l'aspect et de la modalité est prise en charge par d'autres procédés, en particulier l'élaboration de constituants verbaux complexes. L'étude des phénomènes de hiérarchisation et de sérialisation illustre comment, à défaut de posséder les ressources d'une morphologie riche, le samba leko exploite un nombre réduit de morphèmes et comment la mise en discours participe, dans ce type de langue, à la construction du sens.
\end{abstract}

Mots-clés : verbe, aspect, mode, modalité, série verbale, grammaticalisation, Adamawa, NigerCongo

\section{INTRODUCTION}

Le samba leko est une langue tonale de tradition orale parlée au Cameroun et au Nigeria par 10000 personnes selon Fardon (1988). Elle est classée par Greenberg (1966) dans le groupe 2 de la branche Adamawa ${ }^{1}$.

Le propos du présent article est d'exposer comment, au moyen d'un système de conjugaison relativement simple, de l'élaboration de constituants verbaux (CV) complexes et de l'emploi de quelques unités relatives à l'énoncé, cette langue élabore l'expression de l'aspect et du mode. Le système verbal sera d'abord présenté, suivi des CV complexes.

Les unités grammaticales qui seront évoquées dans ce travail, mais dont la portée dépasse le CV, sont :

- $\quad$ l'effectif (Eff.) qui indique la véracité des propos ou l'effectivité du procès ;

\footnotetext{
${ }^{1}$ Cet article repose sur des données recueillies dans un village du Nord-Cameroun lors de trois missions financées par Paris III et le CNRS (LLACAN, UMR 8135). Ces missions n'auraient pu se réaliser sans l'aide de ces institutions, de tous ceux qui m'ont accueillie, de mon assistant et de l'ensemble des villageois que je remercie ici. Cet article reprend et développe une communication présentée à Leiden en août 2001 , lors du $31^{\text {st }}$ CALL. Je remercie également les relecteurs du JALL pour leurs suggestions éclairées.
} 
- le distanciatif (Dist.) qui signale que le lieu du procès est différent de celui de l'énonciation ;

- les particules énonciatives, dont les marques de négation ;

- les modalités d'énoncé neutre (ME neutre) et fréquentative (Fréq.), nécessaires à la bonne formation de l'énoncé assertif ou interrogatif, en l'absence de particule énonciative ${ }^{2}$.

\section{LE SYSTÈME VERBAL}

Le système verbal distingue deux modes (l'assertif et l'obligatif) marqués par des indices personnels sujet différents présentés en Figure $1^{3}$.

\begin{tabular}{|c|c|c|c|c|}
\hline \multicolumn{3}{|c|}{ ASSERTIF } & \multicolumn{2}{|c|}{ OBLIGATIF } \\
\hline & sg. & pl. & sg. & pl. \\
\hline 1 & má & $\begin{array}{l}\text { bá (exc.) } \\
\text { bôn...-ñ (incl.) }\end{array}$ & má & $\begin{array}{l}\text { bá (exc.) } \\
\text { bân ...-ñ (incl.) }\end{array}$ \\
\hline 2 & ń & ?í & ǹ & ?ì \\
\hline 3 & $\varnothing$ & bə̀ & ?ô & bồ \\
\hline $\log$. & bàn & bân...-n̄á & - & - \\
\hline
\end{tabular}

Figure 1 Les indices sujet

Chacun de ces modes dispose d'une forme simple dans laquelle le verbe apparaît dans sa forme lexicale et d'une ou deux formes complexes à auxiliaire dans laquelle la base verbale construit un verbonominal ${ }^{4}$.

\begin{tabular}{lcc}
\hline & ASSERTIF & OBLIGATIF \\
\hline FORME SIMPLE (verbe dans sa forme lexicale) & Absolu & Absolu \\
FORMES À AUXILIAIRE (et à verbonominal) & Progressif & Intensif \\
& Futur & \\
\hline
\end{tabular}

Figure 2 Le système verbal

Le choix d'une forme simple ou d'une forme à auxiliaire s'accompagne d'un changement dans l'ordre des éléments : les énoncés verbaux manifestent l'ordre SVO avec une conjugaison à l'absolu, mais $\mathrm{S}$ Aux. $\mathrm{O}$ VN avec une conjugaison à auxiliaire.

${ }^{2}$ Les abréviations utilisées sont indiquées en fin d'article.

3 Dans le mot à mot des exemples, l'obligatif est signalé par Obl. L'assertif étant considéré comme le mode par défaut, il n'est pas indiqué.

${ }^{4}$ J'oppose le verbonominal $(V N)$, qui se construit sur le modèle [base verbale $+-\overline{\mathbf{n}}$ ] et ne s'emploie que dans un CV conjugué avec un auxiliaire, à l'infinitif qui se construit sur le modèle $[\mathrm{VN}+$ Marque de l'infinitif bè]. La réalisation du dérivatif $-\overline{\mathbf{n}}$ répond à des règles complexes qu'il serait trop long de développer ici (cf. Fabre 2003:162-163).

Je réserve le terme d'auxiliaire aux auxiliaires de conjugaison. Je montrerai que dans les CV complexes, certains verbes s'apparentent à des auxiliaires (quelques-uns s'accordent en transitivité avec le verbe qu'ils déterminent, ils peuvent perdre leur trait (in)transitif et leur sens processif), mais qu'aucun cas de grammaticalisation ne conduit à intégrer ces verbes à la catégorie des auxiliaires de conjugaison. 
$\mathrm{Au}$ mode assertif et en présence d'un sujet lexical, l'emploi de l'indice sujet de $3^{\mathrm{e}}$ personne du pluriel n'est pas nécessaire à la bonne formation de l'énoncé, sauf dans le cas d'un CV sériel (cf. section 3) ${ }^{5}$. La présence de cet indice personnel signale la topicalisation du sujet.

En revanche, l'indice sujet obligatif est nécessairement présent lorsque le CV est conjugué au mode obligatif. Aussi, le mode assertif est le mode le moins marqué formellement.

La langue ne présente donc que deux modes, dont l'un (l'obligatif) est sémantiquement et formellement plus marqué que l'autre (l'assertif). La valeur sémantique de l'assertif est en conséquence relativement neutre ; c'est le mode le plus fréquent dans les textes, celui qui permet à l'énonciateur de situer le procès sur le plan de la réalité affirmée. Les conjugaisons absolues n'impliquent pas un aspect spécifique et recouvrent toutes les valeurs aspectuelles possibles pour ces deux modes. Les formes à auxiliaire sont vraisemblablement plus récentes et permettent d'expliciter certaines valeurs.

\subsection{Le mode assertif}

Le mode assertif est celui de l'assertion, qu'elle soit verbale ou non verbale ${ }^{6}$. L'assertif est aussi le mode qui n'exprime ni ordre ni interdiction. Il rassemble l'assertif absolu, l'assertif progressif et l'assertif futur.

\subsubsection{L'assertif absolu}

L'assertif absolu est la conjugaison simple du mode assertif. Le CV conjugué à l'assertif absolu est constitué d'un indice sujet - qui peut ne pas être présent avec un sujet lexical - et du verbe dans sa forme lexicale. Cette conjugaison présente le procès comme réel et établi, sans le situer dans un temps ou un aspect particulier. C'est ce qu'illustrent les deux traductions françaises proposées pour l'exemple hors contexte (1).

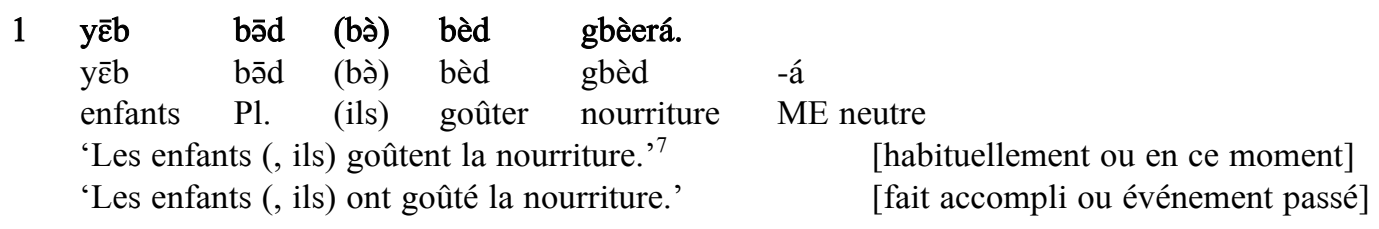

Cette conjugaison a une valeur de vérité générale qui peut être spécifiée par l'emploi de certaines unités. C'est le cas de la modalité d'énoncé fréquentative kú qui, associée à un assertif absolu, confère au procès un caractère répétitif ou habituel, comme dans

${ }^{5}$ L'indice sujet de $3^{\mathrm{e}}$ personne du singulier est zéro pour l'assertif, il est noté $\varnothing$ dans les exemples.

${ }^{6}$ Les indices sujet employés dans les énoncés nominaux sont ceux du mode assertif.

7 La première ligne des exemples rapporte à la notation phonétique. Les lignes suivantes indiquent la notation phonologique et le mot à mot. Sur la réalisation des phonèmes (allongement vocalique dans certains contextes, réalisation [r] de /d/ à l'intervocalique, etc.), on se reportera à Fabre (2003:20-62). 
l'énoncé $(2)^{8}$, ou de l'effectif $i^{9}$ qui, dans ce contexte, présente explicitement le procès comme accompli (3).

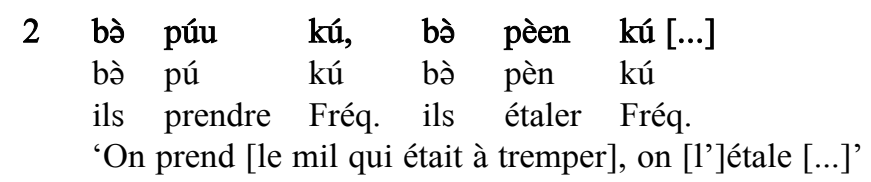

$\begin{array}{llllll}3 & \text { bà líi } & \text { díb } & \text { yă. } & \\ \text { bà } & \text { lí } & \text { díb } & \text { ì } & \text {-á } \\ \text { ils } & \text { manger } & \text { poisson } & \text { Eff. } & \text { ME neutre }\end{array}$

\subsubsection{L'assertif progressif}

L'assertif progressif est une conjugaison complexe qui emploie l'auxiliaire tá et dans laquelle la base verbale construit un verbonominal. Par sa valeur originelle vraisemblablement locative, l'auxiliaire progressif t t $^{10}$ présente le procès dans son déroulement, il localise le sujet par rapport au procès, en quelque sorte au sein du procès $(4)$.
4 yēb bə̄d
yēb bād
enfants Pl. (ils) Prog. nourriture goûter +VN ME neutre
$\begin{array}{lll}\text { (bà) } & \text { tá } & \text { gbèd } \\ \text { (bà) } & \text { tá } & \text { gbèd }\end{array}$
bēerà.
'Les enfants (, ils) sont en train de goûter la nourriture.'

Il est possible d'associer au progressif le distanciatif -à qui indique que le procès se déroule en un lieu éloigné de celui de l'énonciation. Le distanciatif suit alors directement l'auxiliaire progressif. Cette combinaison permet d'exprimer un aspect progressif situé dans le passé. Cet effet de décalage temporel est induit par le temps que l'énonciateur a mis pour parcourir la distance entre le lieu du procès et celui de l'énonciation (5).

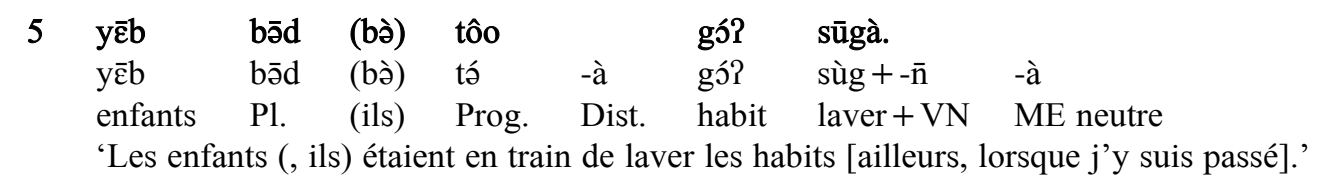

${ }^{8}$ Cet exemple (2) montre en outre que les unités pronominales de $3^{\mathrm{e}}$ personne du pluriel sont employées avec une valeur d'indéfini.

${ }^{9}$ En plus d'une modalité relative à la véracité, l'effectif ì exprime un aspect propre au caractère achevé du procès. C'est ce qui le rend incompatible avec le progressif.

${ }^{10}$ L'auxiliaire du progressif tá et l'actualisateur (ex. 54) tá sont homophones. Leurs distributions respectives et les paradigmes dans lesquels ils s'intègrent me conduisent à considérer qu'il s'agit de deux unités homophones d'origine commune et non d'un élément polyfonctionnel (cf. Fabre 2003:113). 


\subsubsection{L'assertif futur}

L'assertif futur est une conjugaison complexe du mode assertif que l'on identifie par l'association de l'auxiliaire futur dá à un verbonominal. Cet auxiliaire manifeste la grammaticalisation du verbe dá 'aller'. Situant le procès dans un futur plus ou moins proche, cette conjugaison a certaines valeurs virtuelles; il s'emploie aussi parfois pour exprimer un conseil (6).

$\begin{array}{lllllll}6 & \text { ń } \quad \text { dá } & \text { bādn } & \text { ń } & \text { gbāyà. } & \\ \text { ń } & \text { dá } & \text { bādn } & \text { ń } & \text { gbàn }+-\bar{n} & \text {-à } \\ \text { tu } & \text { Fut. } & \text { vin } & \text { ton } & \text { préparer + VN } & \text { ME neutre } \\ \text { 'Tu prépareras ton vin.' } & \end{array}$

Dans des propositions dépendantes, cette conjugaison est attestée associée à l'effectif ì. Le procès posé dans le futur doit alors être effectif et accompli avant que le second procès (celui de la principale) ne commence. On comparera ainsi les énoncés (7) et (8) ci-dessous.

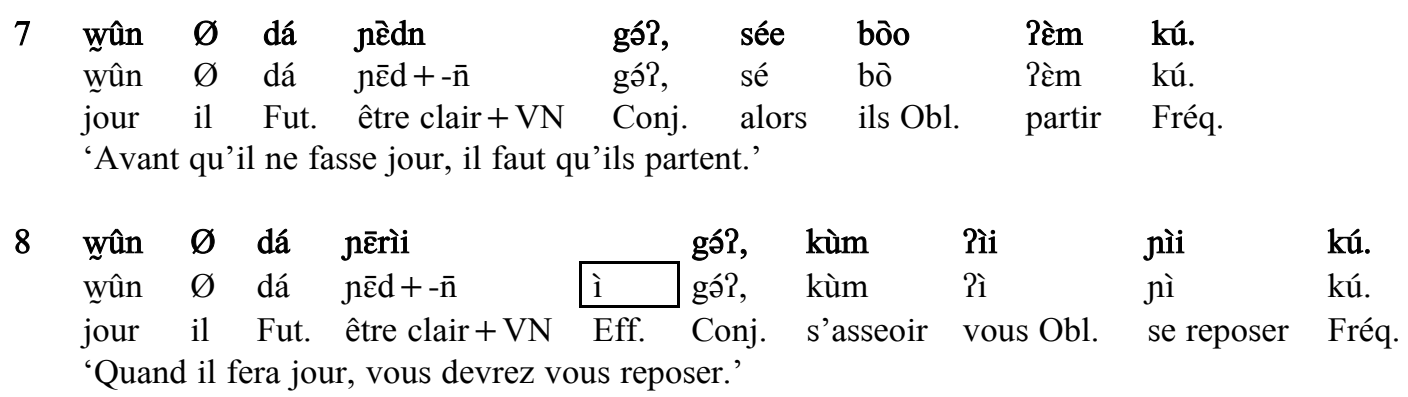

\subsection{Le mode obligatif}

Le mode obligatif est propre à l'énoncé verbal. Formellement, ce mode est marqué par un paradigme spécifique d'indices personnels nécessaires à la bonne formation du CV. C'est le mode de l'injonction, de l'obligation, de l'autorisation ou de l'interdiction. En l'utilisant, le locuteur indique sa volonté que le référent du sujet prenne part au procès - ou n'y prenne pas part dans le cas d'une négation.

Le mode obligatif comprend deux conjugaisons : l'obligatif absolu et l'obligatif intensif.

\subsubsection{L'obligatif absolu}

L'obligatif absolu est une conjugaison simple que l'on identifie par l'emploi de l'indice sujet obligatif précédant le verbe dans sa forme lexicale. De la même façon que l'assertif absolu exprime les valeurs prototypiques du mode assertif, l'obligatif absolu recouvre les différentes valeurs du mode obligatif : injonction, obligation (9), mise en garde et interdiction.

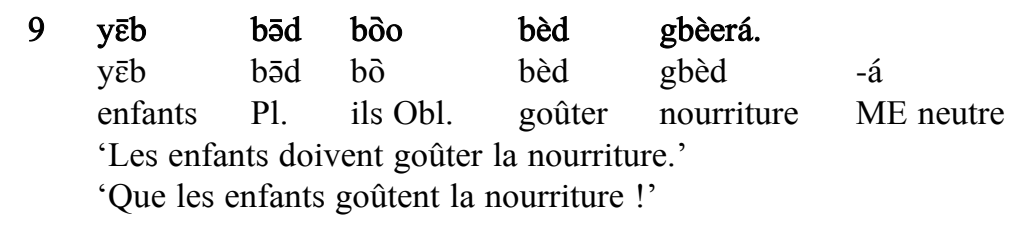




\subsubsection{L'obligatif intensif}

L'obligatif intensif s'identifie par la présence

- de l'indice sujet obligatif,

- d'un verbe suivi du verbonominal dérivé du même verbe,

- d'un ton haut portant sur le verbonominal et, le cas échéant, sur la modalité d'énoncé neutre -à qui est alors réalisée -ā.

Cette conjugaison complexe peur être schématisée par la figure 3.

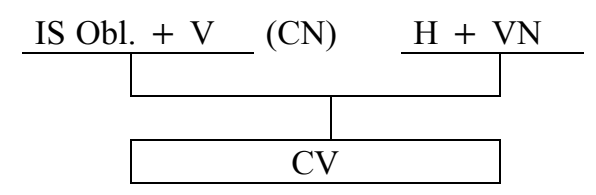

Figure 3 L'obligatif intensif

Cette conjugaison s'emploie pour exprimer un ordre plusieurs fois intimé (réclamation du locuteur), une autorisation qui ne peut être délivrée que suite à un autre procès (10), ou donnée « à contre-cœur », suite aux réclamations de l'interlocuteur.
10 yēb bə̄d bòo j̄̄o
yêेb bād bồ n̄̄
wāə nál nāā.
wāl H jō̄ $+\bar{n}$
$\mathrm{H}$-à
enfants Pl. ils Obl. Int. boire eau Int. boire +VN Int. ME neutre

'Que les enfants boivent de l'eau ! [maintenant que le remède est prêt]'

\subsection{Récapitulatif}

À titre de synthèse sur la conjugaison du samba leko, on peut considérer que ce système relativement simple s'organise autour d'une distinction primaire de mode (assertion et injonction). En dehors du cas du progressif, ce n'est pas le système verbal qui prend en charge l'expression de l'aspect. De nombreuses informations d'ordre aspectuel, temporel et modal s'expriment donc au travers de procédés autres que la conjugaison, notamment, comme on l'a évoqué, l'emploi de différents éléments grammaticaux (dont l'effectif, le distanciatif, les modalités d'énoncé et les particules énonciatives). En outre, l'élaboration de CV complexes sériels ou hiérarchisés participe à l'expression d'aspects et de modalités particuliers.

\section{LES CV COMPLEXES}

Par $C V$ complexe, j'entends un CV composé de plusieurs bases verbales. Le samba leko en présente deux types construits à partir de verbes autonomes : le CV hiérarchisé et le CV sériel (ou série verbale). Cette langue fait grand usage de la série verbale, puisque plus de $41 \%$ des énoncés verbaux spontanés du corpus présentent au moins un CV sériel, alors que moins de $8 \%$ attestent un CV hiérarchisé.

Ces deux types sont structurellement très différents. 


\title{
3.1 Structuration des deux types de CV complexe
}

Le CV hiérarchisé est une structure de contrôle dans laquelle le verbe conjugué régit un groupe infinitif (i.e. un ensemble constitué d'un infinitif et, le cas échéant, d'un constituant nominal). En (11) kò 'saisir' régit le groupe [náb nàanbè] 'danser la danse'"

\author{
11 bò kòo náb nàanbèá. \\ \begin{tabular}{llllll} 
bà & kò & náb & nā $+-\bar{n}$ & bè & -á \\
\cline { 2 - 4 } ils & saisir & danse & danser + VN & Inf. & ME neutre
\end{tabular} \\ 'Ils continuent de danser.'
}

Le CV sériel se caractérise par sa constitution en plusieurs verbes (trois au maximum) et la présence nécessaire de l'indice sujet, quelle que soit la conjugaison choisie. Selon les critères de Durie (1997), la sérialisation en samba leko est de type nucléaire (la conjugaison - marquée en particulier par les indices sujet - n'est exprimée que sur un seul des verbes).

La position particulière des indices contribue à l'identification du CV sériel :

- avec une conjugaison absolue, les indices sujet et complément (et/ou les compléments du prédicat) se trouvent de part et d'autre du dernier verbe de la série (12);

\begin{tabular}{|c|c|c|c|c|c|c|c|c|}
\hline núu & bə̄d & dáa & líi & bə̀ & béc & $y \bar{\varepsilon} d$ & yă. & \\
\hline nú & bə̄d & dá & lí & bà & bé & $y \bar{\varepsilon} d$ & ì & -á \\
\hline oiseau & Pl. & aller & manger & ils & finir & mil & Eff. & ME neutre \\
\hline
\end{tabular}

'Les oiseaux s'en sont allés et ont fini de manger le mil.'

- avec une conjugaison à auxiliaire, les indices sujet et complément (et/ou les compléments du prédicat) se placent de part et d'autre de l'auxiliaire, l'ensemble [indice sujet + auxiliaire] se situant généralement ${ }^{12}$ devant le dernier verbe de la série ${ }^{13}$. Le verbe de la série placé après le groupe [indice sujet + auxiliaire] est régi par l'auxiliaire et construit un verbonominal, ceux qui se trouvent avant ce groupe apparaissent dans leur forme lexicale (première série de l'exemple 13).

11 Sur la relation entre le verbe nā et le nom náb, voir la section consacrée à la composition verbale et aux compléments privilégiés dans Fabre (2003:164-182).

12 Il faut peut-être voir, dans la relative mobilité de l'auxiliaire progressif ou futur au sein de la série verbale, la trace d'un conflit entre le sémantisme de ces conjugaisons (elles présentent le procès en dehors de son accomplissement) et le sémantisme de la construction sérielle qui implique que le procès du verbe principal est acquis.

Le corpus ne présente aucune attestation de série verbale à l'obligatif intensif.

${ }^{13}$ La distinction proposée par Durie (1997:302) entre séquence contigüe (where any argument are placed outside the verb string) et séquence non-contigüe (where arguments can intervene between verbs) est difficilement applicable au samba leko. En effet, on peut considérer que la sérialisation est de type contigu puisque les compléments, qui sont en facteur commun, se placent après le dernier verbe dans le cas d'une série conjuguée à l'absolu (l'énoncé 13 ' *ná gà? wà $\varnothing$ wòb kú, dans lequel le complément gà? wà s'insère entre les deux verbes, est agrammatical). Mais on peut aussi considérer que la sérialisation est de type non-contigu puisque (a) l'indice personnel sujet se place devant le dernier verbe et (b) le(s) complément(s) intervien(nen)t entre l'auxiliaire et le dernier verbe (au verbonominal) dans le cas d'une série verbale conjuguée avec un auxiliaire. 
13 \begin{tabular}{ll|llll} 
tǒl & záa & $\varnothing$ & dá & 1èm & gá?, \\
tǒl & zá & $\varnothing$ & dá & 1èm $+-\bar{n}$ & gá? \\
\cline { 5 - 6 } lièvre & se lever & il & Fut. & partir + VN & Conj.
\end{tabular}

\begin{tabular}{|c|c|c|c|c|}
\hline náa & $\varnothing$ & wòb & gàwàa & \\
\hline ná & $\varnothing$ & wòb & gà? & -wà \\
\hline ne & il & casser & corne & petite \\
\hline
\end{tabular}

'Lièvre s'est levé et, avant de partir, il a piétiné et cassé la petite corne.'

À ma connaissance, la position de l'indice sujet devant le dernier verbe d'une série n'est pas attestée dans d'autres langues SVO ou S Aux O VN ; cet ordonnancement est propre aux langues à verbe final ${ }^{14}$.

Les deux types de constructions complexes ne sont pas exclusifs l'un de l'autre, un même $\mathrm{CV}$ peut manifester à la fois des phénomènes de hiérarchisation et de sérialisation, mais il n'y a jamais qu'un seul verbe conjugué.

En (14) le verbe dá 'aller' fonctionne comme verbe hiérarchisant (il régit nād kòombè) au sein d'une série verbale qu'il construit avec wál 'se réunir'.

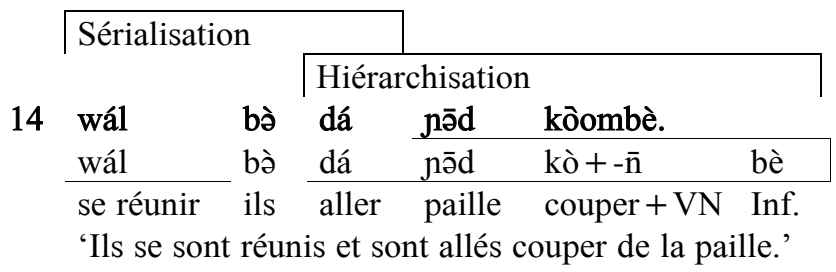

En (15), le verbe 1èm 'partir' régit deux infinitifs. Dans une série verbale à l'infinitif, la marque de l'infinitif bè n'apparaît qu'une seule fois, généralement après la dernière base verbale.

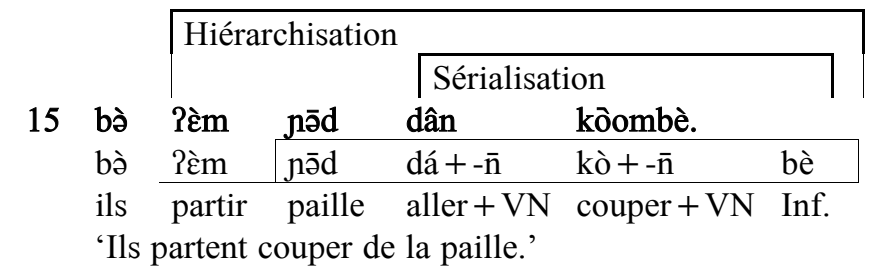

Sur le plan syntaxique, on retiendra surtout que la position des marques de conjugaison, en particulier celle de l'indice sujet, indique que :

- le verbe syntaxiquement marqué ou conjugué (verbe principal) dans le CV hiérarchisé est le premier verbe de la structure ; il est le seul à être conjugué et il régit un groupe infinitif ;

- le verbe syntaxiquement marqué ou conjugué (verbe principal) dans le CV sériel est le dernier verbe de la série ${ }^{15}$.

\footnotetext{
${ }^{14}$ Le propos est ici de rapprocher deux structures fort différentes et de les confronter afin de mettre en évidence les effets de sens qu'elles induisent. Une étude typologique de la sérialisation en samba leko ferait l'objet de nouvelles enquêtes et d'une autre publication. De nombreux travaux ont été publiés sur ce thème (cf. notamment Delplanque (1998), Durie (1997) ou Lord (1993) et les bibliographies données dans ces travaux).
} 


\subsection{Sémantisme des deux types de CV complexe}

Si ces constructions s'opposent sur le plan structurel, les effets de sens qu'elles produisent sont relativement parallèles. Dans les deux constructions, soit les différents verbes conservent leur sens processif, soit le verbe principal tend à perdre sa valeur processive au profit d'une valeur plus abstraite, aspectuelle ou modale.

\subsubsection{Les CV complexes à valeur processive}

Lorsque les verbes conservent leur sens processif, l'ordonnancement des différentes bases verbales correspond à la chronologie des procès de ces verbes. Le prédicat exprime un processus au sein duquel les différents procès sont présentés de façon différente selon que le CV est hiérarchisé ou sériel.

- Le CV hiérarchisé met en lumière le procès de départ du processus (le verbe conjugué est le premier verbe). Le procès du verbe infinitif est considéré comme visé mais non acquis (cf. 18 par exemple).

- Le CV sériel relate en plusieurs procès un événement unique, et met en lumière le procès d'arrivée (le verbe conjugué est le dernier verbe) qui est présenté comme atteint et acquis. Les autres verbes de la série retracent rétrospectivement le moyen ou le cheminement qui a abouti à ce procès (cf. 19 ou 20 par exemple).

\subsubsection{Les CV complexes à valeur aspecto-modale}

Lorsque le verbe principal perd sa valeur strictement processive au profit d'une valeur plus abstraite (aspectuelle ou modale), l'ordonnancement des différentes bases verbales n'est pas non plus anodin. La modalité ou l'aspect qu'exprime le verbe principal a une portée :

- prospective dans le cas du $\mathrm{CV}$ hiérarchisé : $\mathrm{V}_{1}$ régit $\mathrm{V}_{2}$ (qui est un infinitif) et le procès de $\mathrm{V}_{1}$ est chronologiquement antérieur à celui de $\mathrm{V}_{2}$. Ce sont des modalités qui anticipent le procès qu'elles déterminent, elles relèvent souvent du déontique volitif ou potentiel (vouloir, souhait, aptitude à réaliser le procès) et impliquent une visée. Les aspects prospectifs soulignent le caractère initiatif ou duratif du procès ;

- rétrospective dans la série verbale $: \mathrm{V}_{2}$ détermine $\mathrm{V}_{1}$ et le procès de $\mathrm{V}_{2}$ est chronologiquement postérieur à celui de $\mathrm{V}_{1}$. Ce sont principalement des modalités validantes (modalités appréciatives liées à la validation du procès ou à la validation d'une capacité à le réaliser) et des aspects relatifs au résultat du procès, à son caractère achevé, répétitif ou habituel.

D'une certaine façon, le verbe principal d'un CV hiérarchisé est susceptible de véhiculer une modalité a priori ou un aspect relatif au prélude du procès déterminé, alors que le verbe principal d'une série est susceptible de véhiculer une modalité $a$ posteriori ou un aspect relatif à l'approche du terme du procès déterminé.

\footnotetext{
15 Parce qu'il est l'unique verbe conjugué, je considère que le dernier verbe de la série est le verbe principal. Néanmoins, cette dénomination ne doit pas être comprise dans le sens de verbe régissant. Le seul $\mathrm{CV}$ complexe qui manifeste une dépendance entre les différentes bases verbales est le $\mathrm{CV}$ hiérarchisé. La base verbale régie y construit d'ailleurs un infinitif, et l'infinitif s'élabore à partir du verbonominal qui est la forme verbale dépendante par excellence.
} 


\subsubsection{Récapitulatif}

Dans cette présentation du CV complexe, les verbes principaux du CV hiérarchisé et du $\mathrm{CV}$ sériel seront respectivement appelés verbes prospectifs et verbes rétrospectifs. Ces dénominations n'ont de sens que dans le cadre du CV complexe, elles recouvrent à la fois la position du verbe principal par rapport aux autres bases verbales du CV et la valeur aspectuelle ou modale impliquée par ces verbes.

La figure 4 schématise les deux CV complexes, sur les plans formel et sémantique. Au sein de chaque structure, le verbe en gras est le verbe principal, celui sur lequel se cristallisent les marques de conjugaison.

\begin{tabular}{|c|c|c|}
\hline TYPE SYNTAXIQUE & CV HIÉRARCHISÉ & CV SÉRIEL ${ }^{16}$ \\
\hline TYPE SÉMANTIQUE & $\mathrm{V}_{\mathrm{inf}}$ & $\mathrm{V}_{1} \ldots \mathrm{V}_{\mathrm{n}}$ \\
\hline CV à valeur processive & \multicolumn{2}{|c|}{$\begin{array}{l}\text { Processus avec mise en lumière du procès } \\
\text { de départ } \\
\text { d'arrivée }\end{array}$} \\
\hline CV à dimension aspecto-modale & $\begin{array}{c}\mathrm{V}_{1}: \text { aspect } / \text { modalité de sens } \\
\text { PROSPECTIF }\end{array}$ & $\begin{array}{c}\mathrm{V}_{\mathrm{n}} \text { : aspect/modalité de sens } \\
\text { RÉTROSPECTIF }\end{array}$ \\
\hline
\end{tabular}

Figure 4 Les CV complexes

\subsection{Emplois des CV complexes}

Je présenterai d'abord des occurrences de CV complexes à valeur processive, puis les CV complexes à valeur aspecto-modale. À partir d'exemples, j'entends maintenant montrer comment la langue (a) puise dans le sémantisme de certains verbes pour exprimer tantôt un procès, tantôt un aspect ou une modalité et (b) exploite le choix entre les deux structures comme pour suppléer au faible développement de son système verbal.

Dans le mot à mot des exemples, le parti est pris de :

- conserver le sens processif du verbe (celui qu'il a en général dans un CV simple) lorsque le sens qu'il prend en tant que verbe principal dans un $\mathrm{CV}$ complexe est jugé proche de ce sens ;

- proposer plusieurs traductions pour un même verbe dont le sens varie fortement d'une construction à l'autre.

\subsubsection{Les CV complexes à valeur processive}

Les CV sériels à valeurs processive ont été largement décrits dans la littérature et les attestations en samba leko coïncident bien avec ces études de la sérialisation.

Dans les CV sériels à valeur processive, les verbes s'ordonnent selon la chronologie des procès et le prédicat a une valeur cumulative qui correspond à la somme des procès des verbes en présence. Les associations entre les différents verbes peuvent être

\footnotetext{
${ }^{16}$ Dans la figure $4, V_{n}$ désigne le dernier verbe de la série. Dans le corpus, celle-ci est constituée
} de deux ou de trois verbes. 
considérés comme libres, si tant est que les différents procès puissent s'associer au sein d'un événement unique (ex. 'chercher-trouver' dans l'exemple 16).

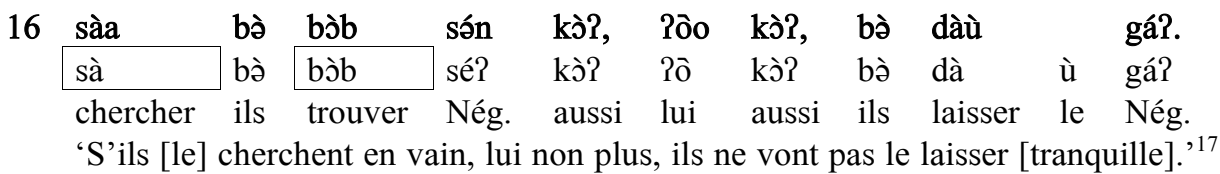

Qu'il s'agisse des CV sériels ou des CV hiérarchisés, les premiers verbes sont bien souvent des verbes de déplacement $(12,15,18,19$ ou 20).

Le verbe dá 'aller' illustre le fonctionnement des verbes qui expriment un procès de déplacement. Ce verbe est susceptible d'être employé dans un CV simple (17).

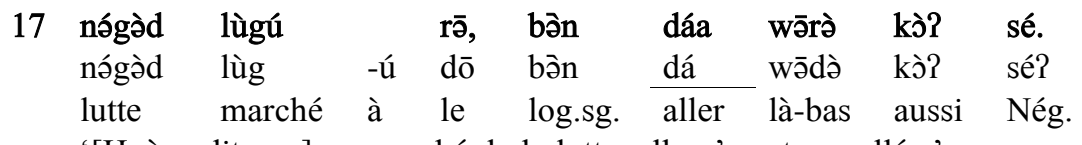

'[Hyène dit que] au marché de la lutte, elle n'y est pas allée.'

Le verbe dá 'aller' est susceptible de fonctionner comme verbe prospectif au sein d'un CV hiérarchisé (18). Cette construction induit que le procès qu'exprime dá a pour visée ou finalité particulière le procès du verbe régi.
18 Ríi dáa yēd pàambòá.
lí dá \begin{tabular}{lllll}
\cline { 3 - 4 } & pà $+-\bar{n}$ & bè & -á
\end{tabular}
vous aller mil prendre+VN Inf. ME neutre
'Vous êtes partis [pour] prendre le mil.'

Le verbe dá 'aller' peut aussi être employé dans une série verbale. Le plus souvent, il intervient en première position dans la série - i.e. pas comme « verbe principal »- $(12$, $19,20)$.

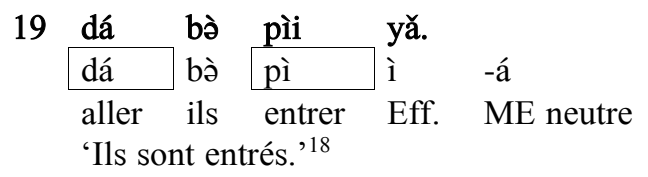

${ }^{17}$ La série verbale relate un événement unique constitué de différents procès. Lorsque la proposition comporte une négation, celle-ci porte sur l'ensemble du prédicat ('chercher-trouver' dans l'exemple 16) et non sur le seul dernier verbe. Dans l'exemple (16), il est impossible de considérer que la négation est distribuée sur chacun des deux verbes $(*$ 'ils ne cherchent pas ne trouvent pas') puisque, dans le contexte de cette occurence, les personnages cherchent activement. Un énoncé relatant plusieurs événements avec une polarité différente comme 'Ils cherchent mais ne trouvent pas' se traduit nécessairement par une succession de propositions exprimant chacune un événement, chacun d'eux pouvant être nié « individuellement » : bà sà kú ; bà bòb sé?.

${ }^{18}$ On sait qu'une série verbale peut se traduire par un seul verbe dans les langues qui, comme le français, ignorent ce type de construction. C'est le cas dans l'exemple (19). L'exemple (19') bà pì yă est aussi attesté pour traduire 'Ils sont entrés', et je ne suis pas en mesure de dire quelle est la différence entre les énoncés (19) et (19'). Je signale toutefois qu'il semble plus fréquent, dans les pièces littéraires qui constituent le corpus, d'employer conjointement les deux verbes que d'employer le seul verbe pì. 


\begin{tabular}{|c|c|c|c|c|c|c|c|c|}
\hline dáa & $\varnothing$ & sàa & Pīn & bārāā & & bóonú & & \\
\hline dá & $\varnothing$ & sà & & bə̄d & $-\overline{\mathrm{a}}$ & & -ú & \\
\hline all & il & chercher & chose & Pl. & ces (Rel.) & grenier & dans & le (Rel.) \\
\hline
\end{tabular}

Dans les deux types de constructions complexes, le prédicat exprimé correspond à la somme des différents procès. La confrontation des énoncés $(18)$ et $(19,20)$ montre que la construction hiérarchisée implique une visée (18) que n'implique pas la construction sérielle $(19,20)$.

\subsubsection{Les CV complexes à valeur aspecto-modale}

Les verbes principaux des CV sériels et des CV hiérarchisés ont un fonctionnement autonome dans les CV simples. La modalité ou l'aspect qu'ils expriment dans les CV complexes est toujours lié à leur sens dans les CV simples.

En effet, la plupart des verbes attestés comme verbes principaux à valeur aspectomodale ont déjà, au niveau du lexique, un sens modal ou aspectuel que l'on peut qualifier de prospectif ou de rétrospectif.

Ainsi, les verbes attestés comme verbes principaux à valeur aspectuelle ou modale dans les CV hiérarchisés (verbes prospectifs) renvoient souvent, lorsqu'ils construisent des CV simples, à des procès non bornés ('courir', 'vouloir') ou intrinsèquement inceptifs ('entrer', 'tomber').

A l'inverse, les verbes principaux à valeur aspectuelle ou modale dans les séries verbales (verbes rétrospectifs) expriment souvent, lorsqu'ils construisent des CV simples, des procès tournés vers l'achèvement ou le résultat ('réunir', 'réparer', 'finir', 'suffire').

Aussi, pour certains de ces verbes, la distinction sémantique que je propose (CV complexe à valeur processive versus $\mathrm{CV}$ complexe à valeur aspecto-modale) est parfois difficile maintenir.

La figure 5 recense les verbes les plus fréquemment attestés comme verbes principaux dans des CV complexes à valeur aspectuelle ou modale. Dans cette figure, le signe indique que le verbe de la première colonne est attesté dans un CV sériel, mais qu'il garde son sens processif. Le signe $\varnothing$ signale que le verbe de la colonne de gauche n'est pas attesté comme verbe principal dans la construction considérée.

Parmi ces verbes, trois seulement n'ont pas une valeur lexicalement prospective ou rétrospective. Ce sont les verbes en grisé dans la figure 5. Cette caractéristique leur permet de fonctionner tantôt comme verbe prospectif, tantôt comme verbe rétrospectif. La confrontation de différentes positions syntaxiques qu'occupent ces verbes au sein des CV complexes mettra en évidence les implications sémantiques des deux structures. 


\begin{tabular}{|c|c|c|c|c|}
\hline $\begin{array}{c}\text { CV SIMPLE } \\
\text { (SENS PROCESSIF) }\end{array}$ & \multicolumn{2}{|c|}{$\begin{array}{l}\text { CV HIÉRARCHISÉ : } \\
\text { V PROSPECTIF }\end{array}$} & \multicolumn{2}{|c|}{$\begin{array}{c}\text { CV SÉRIEL : } \\
\text { V RÉTROSPECTIF }\end{array}$} \\
\hline kò saisir & continuer à & Asp. duratif & & - \\
\hline pì entrer & commencer à & & & - \\
\hline pí tomber & commencer à & Asp. initiatif & & - \\
\hline sód commencer & se mettre à & & & - \\
\hline Pì vouloir, aimer & vouloir & Mod ulitived $^{19}$ & & $\varnothing$ \\
\hline zè détester & ne pas vouloir & Hide. votitites & & $\varnothing$ \\
\hline mòm désigner & faillir & Mod. approximative & & - \\
\hline bíd, bí rentrer & revenir faire & Déplacement motivé & $\begin{array}{l}\text { faire à nouveau / } \\
\text { en retour }\end{array}$ & Asp. itératif \\
\hline dón suffire, équivaloir & avoir le droit de & Mod. permissive & $\begin{array}{l}\text { égaler / faire } \\
\text { intégralement }\end{array}$ & $\begin{array}{l}\text { Mod. équivaleur et } \\
\text { distribution }\end{array}$ \\
\hline bán savoir, rassembler & être capable de & $\begin{array}{l}\text { Mod. potentielle } \\
\text { virtuelle }\end{array}$ & réussir & $\begin{array}{l}\text { Mod. potentielle } \\
\text { validée }\end{array}$ \\
\hline kùd réparer, arranger & & $\varnothing$ & bien faire & Mod. qualitative \\
\hline dì se tenir & & $\varnothing$ & agir sans raison & \\
\hline sá avoir l'habitude de & & $\varnothing$ & avoir l'habitude de & Asp. habituel \\
\hline béd, bé finir & & $\varnothing$ & finir & \\
\hline sày être distribué & & $\varnothing$ & finir et distribuer & \\
\hline
\end{tabular}

Figure 5 Verbes prospectifs et verbes rétrospectifs à valeur aspecto-modale

Ces quinze verbes permettent de montrer comment le sémantisme du verbe et le sémantisme de la construction s'articulent en samba leko pour exprimer certains aspects et certaines modalités.

Les verbes qui participent à l'expression de l'aspect puis de la modalité seront successivement présentés. Dans chacune de ces sections, les verbes prospectifs seront d'abord étudiés, puis les verbes rétrospectifs et enfin les verbes prospectifs et rétrospectifs. On verra le fonctionnement de chacun de ces verbes, d'abord en tant que verbe unique dans un CV simple afin de mettre en évidence son sens processif, puis en tant que verbe principal (prospectif et/ou rétrospectif) dans un CV complexe.

\subsubsection{Expression de l'aspect}

Le duratif et les aspects initiatifs (j'entends par ce terme les différents aspects relatifs au commencement du procès) s'expriment au travers de verbes prospectifs dans des CV hiérarchisés.

L'itératif et l'habituel sont pris en charge par des verbes rétrospectifs au sein de séries verbales.

\section{Les verbes prospectifs}

- Kò 'saisir', aspect duratif

Lorsqu'il construit un CV simple, le verbe kò signifie 'attraper un animal, retenir quelqu'un dans sa course' (21).

19 Les deux verbes considérés ont à la fois une valeur modale volitive (au sens large) et une valeur de sentiment ('aimer', 'détester'). 
21 bò kòo líirō yă.

bò kò lídō ì -á

ils saisir voleur Eff. ME neutre

'Ils ont attrapé le voleur.'

En tant que verbe prospectif dans un CV hiérarchisé, kò prend une valeur aspectuelle qui souligne la durée du procès, comme s'il était maintenu dans son déroulement (11), (22) ou (23).

22 bə̀ dàa gbōgám ì,

bò dà gbāgóm ì

ils laisser bègue Eff.

$\varnothing$ tó kôon wू๊̃̄̄ dêdnbèá.

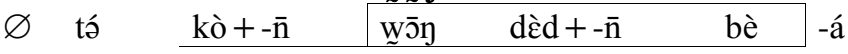

il Prog. durer+VN parole appeler $+\mathrm{VN}$ Inf. ME neutre

'Ils ont abandonné le bègue, il ne cesse d'appeler.' ${ }^{20}$

23 sée $\varnothing$ kòo gàawàā Pîlbèá.

\begin{tabular}{llllllll|l} 
sé & $\varnothing$ & kò & gà? & -wà & $-\bar{a}$ & Píl+-ñ & bè & -á \\
\cline { 3 - 6 } alors & il & durer & corne & petit & cette & siffler + VN & Inf. ME neutre
\end{tabular}

'Alors, il [Lièvre] continue à siffler [avec] cette petite corne.'

- Pì 'entrer', pí 'tomber' et sód 'commencer, se mettre à', aspects initiatifs

Deux verbes formellement et sémantiquement proches sont susceptibles de construire des CV simples et des CV hiérarchisés : pí 'tomber' (24) et pì 'entrer' $(25)^{21}$.

Les exemples (24) et (25) illustrent le fonctionnement de ces verbes dans les CV simples.

24 mó píi wālú.

mó pí wōl -ú

je tomber eau dans

'Je suis tombé dans l'eau.'

25 gbǎl $\varnothing$ pìà kò bòorá.

gbǎl $\varnothing \quad$ pì -à kò bòd -á

hyène elle entrer Dist. avec derrière ME neutre

'Hyène est entrée de dos.'

Le verbe pí 'tomber' se construit le plus souvent de façon intransitive. Le seul complément direct avec lequel ce verbe est attesté est nógə̀d 'lutte, bagarre' qui lui confêre un sens initiatif $(26)^{22}$.

${ }^{20}$ Dans l'énoncé (22), le verbe kò est régi par l'auxiliaire du progressif. C'est la raison pour laquelle il construit un verbonominal. Ce verbe kò régit à son tour l'infinitif dèdnbè.

${ }^{21}$ Ces deux verbes sont vraisemblablement dans un rapport de dérivation, pí 'tomber' pourrait être le dérivé factitif de pì 'entrer'.

22 À propos des verbes à complément privilégié, voir Fabre (2003:164-182). 


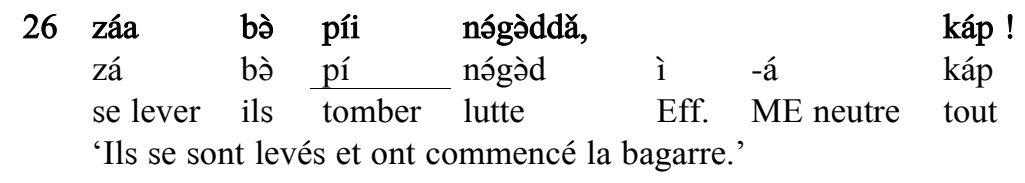

Le verbe sód 'commencer' est lui aussi susceptible de construire un CV simple (27).

\begin{tabular}{llllll}
27 & bòo & sód & nágòd & dā & kú nú ! \\
bồ & sód & nágàd & dō & kú nú \\
\cline { 2 - 3 } & ils Obl. & commencer & lutte & la & Fréq.
\end{tabular}

'Qu'ils commencent la bagarre !'

Lorsqu'ils fonctionnent comme verbes prospectifs dans des CV hiérarchisés, pì 'entrer', pí 'tomber' et sód 'commencer' expriment un aspect initiatif. Dans ce fonctionnement, pì 'entrer' et sód 'commencer' sont plus fréquemment employés (respectivement six et cinq occurrences) que pí 'tomber' (une occurrence).

Les énoncés (28) et (29) présentent l'emploi de pì 'entrer' en tant que verbe prospectif dans un CV hiérarchiséé 23 .
28 tว̀o ń pìi ì nûnbè bóo [...]
tồ ń pì ì nù + Fact. $+-\bar{n}$ bè bó
bon tu entrer Eff. faire courir+VN Inf. aussi
'Bon, tu commences à le faire courir [...]'
29 àlê bə̀sə̀wà
àlê bàsò -wà
allez crapaud petit

Pòo pìi bə̄nó gàwàa Pîlbèá.

$\begin{array}{llllllll}\text { Pô } & \text { pì } & \text { bə̄nó } & \text { gà? } & \text {-wà } & \text { Píl }+-\bar{n} & \text { bè } & \text {-á }\end{array}$

il Obl. entrer log.Pl. corne petite siffler+VN Inf. ME neutre

'Bon, [les ombrettes demandent que] Petit Crapaud commence à leur jouer de la petite corne.'

La seule attestation de pí 'tomber' comme verbe prospectif dans un CV hiérarchisé (30) succède directement et répond à une occurrence de pì 'entrer' dans la même construction (29).
30 bə̀sə̀wà dá $\varnothing$ píi bà gàwàa îllbèá.

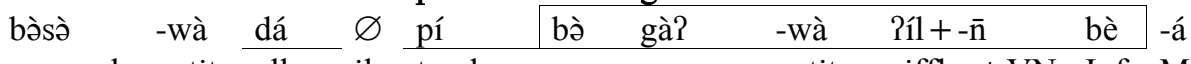
crapaud petit aller il tomber eux corne petite siffler+VN Inf. ME neutre
'Petit Crapaud a commencé à leur jouer de la petite corne.'

L'énoncé (31) illustre l'emploi du verbe sód comme verbe prospectif dans un CV hiérarchisé.

23 Dans ces attestations, pì 'entrer' est en concurrence avec bādîn 'être proche' emprunté au fulfulde 6ad - Noye (1989:51). 
31 ?ồo

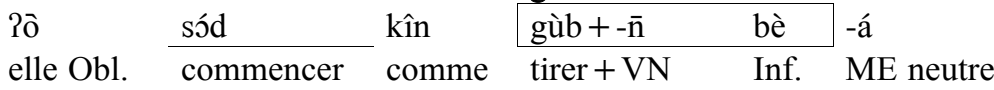

'Qu'elle se mette à tirer!'

Les données dont je dispose actuellement ne me permettent pas d'aller plus avant dans

l'analyse sémantique de ces trois verbes.

\section{Les verbes rétrospectifs}

- Sá 'avoir l'habitude', aspect habituel

Le verbe sá est principalement employé comme verbe rétrospectif dans les séries verbales. Il manifeste le caractère habituel du procès de $\mathrm{V}_{1}(32)$ et (33).

32

\begin{tabular}{|c|c|c|c|c|c|}
\hline gə́ə & Pí & sáa & w్̃̄̄gว̀ & lèe & gú ? \\
\hline gá & Pí & sá & wōgว̀ & lè & gú \\
\hline préparer & vous & avoir l'habitude & sauce & comment & Interro. \\
\hline
\end{tabular}

$33 \quad \begin{array}{lll}\text { in } & \text { bārā } & \\ \text { 2īn } & \text { bād } & -\bar{a}\end{array}$

chose Pl. ces (Rel.) manger elles avoir l'habitude herbe les (Rel.)

$\begin{array}{llll}\text { bà } & \text { yàa } & & \text { sé?. } \\ \text { bò } & \text { yā } & \text {-à } & \text { sé? } \\ \text { elles } & \text { venir } & \text { Dist. } & \text { Nég. }\end{array}$

'Les herbivores ne sont pas venus.'

(Littéralement: 'Les choses qui ont l'habitude de manger de l'herbe, elles ne sont pas venues.')

La seule attestation de sá construisant un CV simple (34) est très proche de (33) cidessus ; ces deux énoncés sont extraits du même conte. Dans ces deux attestations, sá intervient conjointement avec le verbe fù 'manger' dans une proposition relative.

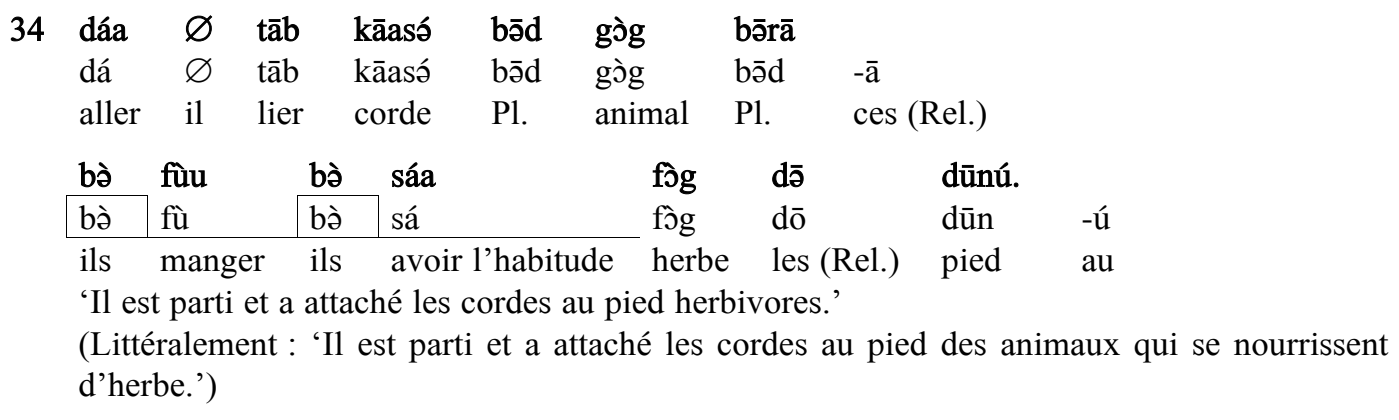

L'énoncé (34) m'a été livré spontanément dans un conte, mais une hésitation du narrateur est perceptible. Dans la mesure où l'informateur de référence l'a répété et confirmé, je le considère comme valide, mais il soulève de nombreuses questions, et c'est la seule attestation de ce type dans le corpus.

Le constituant fôg, qu'il semble logique d'interpréter comme le complément de fû, se place ici après sá. C'est la position habituelle du complément mis en facteur commun dans une série verbale - cf. (33) par exemple.

La présence d'un indice sujet devant chacun des verbes indique que ces verbes ne 
construisent pas une série verbale en (34) du type de celles que l'on l'observe ailleurs dans le corpus ${ }^{24}$.

- Béd, bé 'finir', achèvement

Le verbe bé est le dérivé agentif (et transitif) de béd (intransitif) ${ }^{25}$. Dans des CV simples, ces verbes se construisent de la façon suivante :

35 súsū béd yă.

súsū béd ì -á

conte finir Eff. ME neutre

'Le conte est fini.'

36 má bée tùu yă.

má bé tù? ì -á

je finir travail Eff. ME neutre

'J'ai terminé le travail.'

Employés comme verbes rétrospectifs, les verbes bé et béd marquent l'achèvement ou la complétude du procès des autres verbes de la série. Ces verbes s'accordent en transitivité avec le verbe qu'ils déterminent. Lorsque le verbe déterminé est intransitif, c'est béd qui est employé (37), lorsqu'il est transitif, c'est son dérivé transitif bé qui intervient (12) et (38).

37 yēbā

$\begin{array}{llllllll}\text { yểb } & -\bar{a} & \text { nāa } & \text { bà } & \text { béd } & \text { kân } & \text { kóolé } \\ \text { enfants } & \text { ces } & \text { monter } & \text { ils } & \text { finir } & \text { vraiment } & \text { tous }\end{array}$

'Ces enfants sont tous montés.'

38 zógò? zógò?, nèd léc $\varnothing$ béc līn bād kăn ság!

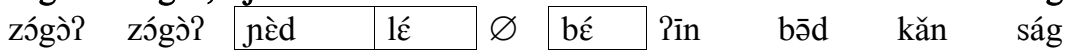

peu peu enlever jeter il finir chose Pl. ainsi tout

'[Il continue de jeter les choses] petit à petit, il a fini de jeter les objets.'

- Sày 'être distribué, achèvement et distribution

Le verbe sàn est un verbe transitif qui exprime une distribution complète et sans reste. L'énoncé (39) présente l'emploi de sầ dans un CV simple.

39 ?īnā

Tīn $\quad-\bar{a} \quad \begin{array}{llll}\text { sầy } & y \bar{e} b & \text { bād } & \text { ì }\end{array}$

chose cette être distribué enfants Pl. Eff. ME neutre

'Cette chose a été intégralement distribuée aux enfants.'

${ }^{24}$ À partir du corpus dont je dispose aujourd'hui, je ne suis pas en mesure de dire si cette construction permet d'exprimer une nuance sémantique particulière ; la traduction est identique pour 33 et 34, mais il conviendrait d'examiner plus précisément cette apparente identité de sens. De nouvelles enquêtes seront aussi nécessaires pour vérifier si cette attestation spécifique (34) doit être analysée comme une variation libre de la série attestée en (33), si d'autres séries $\left[\mathrm{V}_{1} \ldots\right.$ IS $\mathrm{V}_{\mathrm{n}}$ ] admettent une forme [IS $\mathrm{V}_{1} \ldots$ IS $\mathrm{V}_{\mathrm{n}}$ ] et, si tel est le cas, si certains verbes rétrospectifs structurent exclusivement ou librement des séries d'un type ou de l'autre.

${ }^{25}$ Sur la dérivation agentive, voir Fabre (2003:144-145). 
qu'ils ne font pas eux-mêmes le trajet (versus le sens de ce verbe en 42 ci-dessus), mais qu'ils chassent les petits afin que ceux-ci retournent seuls chez eux ${ }^{26}$.

\begin{tabular}{|c|c|c|c|c|c|c|}
\hline nìn & bò & bíà & & kūvêl & bə̄d & kú. \\
\hline nìn & bà & bí & -à & kūvêl & bə̄d & kú \\
\hline & ils & rentr & Dist. & frère & Pl. & Fréq \\
\hline
\end{tabular}

\subsubsection{Expression de modalités}

Les constructions hiérarchisées à verbe prospectif prennent aussi en charge l'expression des modalités volitives ('vouloir' et 'ne pas vouloir, refuser, détester') et de la modalité approximative ('faillir').

L'expression d'une appréciation implique logiquement que le procès considéré est achevé. La modalité appréciative qualitative 'avoir bien fait' relève donc d'une construction sérielle à verbe rétrospectif.

Les modalités déontiques potentielles se formulent tantôt par une construction à verbe prospectif, tantôt par une construction à verbe rétrospectif, selon que l'on exprime une capacité virtuelle ou validée.

\section{Les verbes prospectifs}

- ?î 'vouloir, aimer, désirer' et zè 'se refuser à, détester', modalités volitives

Le verbe ?í 'vouloir, aimer, désirer' est un verbe transitif susceptible de constituer des CV simples comme des CV hiérarchisés. L'énoncé (45) illustre ces deux constructions.

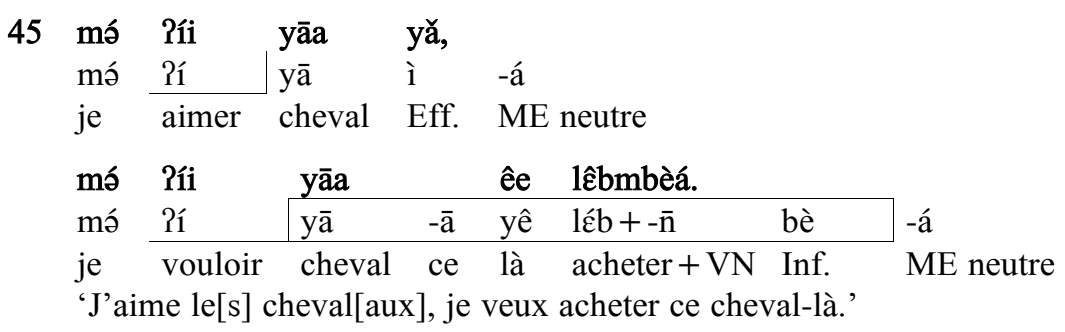

Dans le corpus, le verbe zè 'détester' est principalement employé comme verbe prospectif exprimant une modalité de non-vouloir.

L'exemple (46) présente deux occurrences de ce verbe :

- la première occurrence de zè apparaît dans un CV simple enchâssé au sein d'une proposition relative, Rīn 'chose' est le nom déterminé par la relative et le complément de zè ;

- dans la deuxième occurrence, zè fonctionne comme verbe prospectif dans un CV hiérarchisé.

\footnotetext{
${ }^{26}$ La même série verbale (nìn bí 'chasser rentrer') est attestée dans un autre texte et montre que, dans un autre contexte, le verbe bí 'rentrer' pourra s'interpréter avec un sens processif ('ils chassent et raccompagnent leurs petits frères').
} 


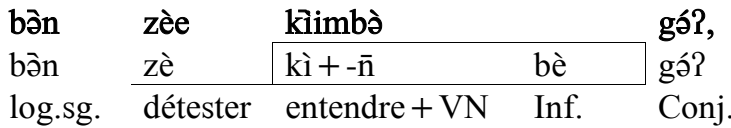

gbăalá, ń tó nîi lūmì ?

gbǎl -á ń tó nî lùm $+-\bar{n}$ ì

hyène ME neutre tu Prog. quoi croquer + VN Interro.

'Hyène [dit que] elle, ce qu'elle déteste, [...] elle ne veut pas entendre : « Hyène, qu'es-tu en train de croquer?»'

- Mòm 'désigner', modalité approximative

Le verbe mòm se construit régulièrement avec nên 'bras' ou nèn yíl 'doigt' dans les CV simples. Il signifie alors 'désigner, montrer ou pointer du doigt' (47).

47 sée záa mòm gbălnìg nēnà yúlìi nûurú. sé zá mòm gbălyìg nề -wà yíl ì nû dú alors se lever pointer lion main petit doigt Eff. œil dans 'Alors [Hyène] s'est levée et a pointé du doigt Lion dans les yeux.'

Fonctionnant comme verbe prospectif, mòm traduit 'faillir', dans le sens d'avoir été sur le point d'arriver sans que cela ait eu lieu (48).

48 bùm $\varnothing$ mòm tàdnù sùdnbèá.

bùm $\varnothing \quad$ mòm $\begin{array}{llll}\text { tàdnù } & \text { sùd }+-\bar{n} & \text { bè }\end{array}$

guerre elle faillir Balkossa pousser+VN Inf. ME neutre

'La guerre a failli repousser [les gens de] Balkossa.'

La valeur 'faillir' suggère que mòm signifie plus précisément 'pointer (vers), viser à, tendre (vers) quelque chose sans (le) toucher, sans (l')atteindre'.

\section{Les verbes rétrospectifs}

- Kùd 'arranger, réparer', appréciation qualitative

Dans une construction simple, le verbe kùd signifie 'arranger, réparer' ou 'fabriquer' (49).

$$
\begin{aligned}
& 49 \text { má kùd sāará. } \\
& \text { mó kùd sād -á } \\
& \text { je fabriquer filet ME neutre } \\
& \text { 'Je fabrique [ou répare] le filet de chasse.' }
\end{aligned}
$$

Fonctionnant comme verbe rétrospectif dans une série verbale, kùd véhicule une modalité appréciative relative au procès de $\mathrm{V}_{1}$ et/ou à son résultat, signifiant que le procès s'est bien déroulé ou que son résultat est conforme et satisfaisant (50).

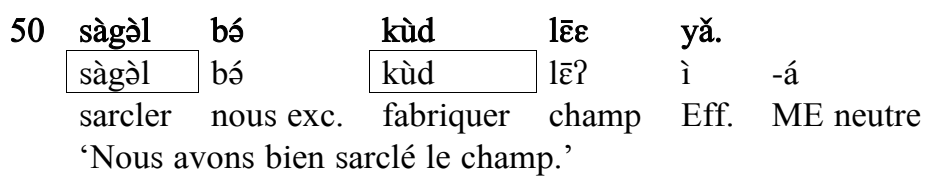


- Dì 'se tenir', gratuité du procès

Le verbe dì est un verbe intransitif qui, employé dans un CV simple, signifie 'se tenir, rester là', voire 'cesser momentanément ses occupations' (51).

$$
\begin{array}{llll}
51 & \text { bà } & \text { dìi } & \multicolumn{1}{c}{\text { kòr. }} \\
& \text { bà } & \text { dì } & \text { kò? } \\
& \text { ils } & \text { se tenir aussi } & \text { auss } \\
& \text { 'Ils ont cessé } & \text { [de jouer].' }
\end{array}
$$

\begin{tabular}{|c|c|c|c|c|c|}
\hline dá & bə̀ & dìi & yă, & & bふón \\
\hline dá & bà & dì & ì & -á & \\
\hline aller & ils & se tenir & Eff. & ME neutre & grenier \\
\hline
\end{tabular}

Lorsque dì fonctionne comme verbe rétrospectif dans une série verbale, il conserve souvent son sens processif (52).

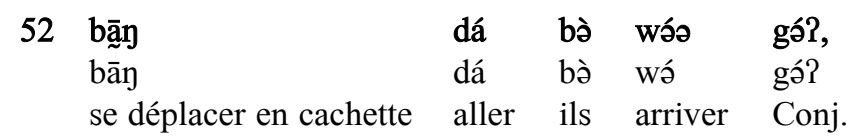

'Ils ont marché en cachette, une fois arrivés, ils sont allés se tenir en bas du grenier.'

Mais, fonctionnant comme verbe rétrospectif, dì peut aussi indiquer le caractère gratuit et non motivé du procès de $V_{1}(53)$. Cet emploi est relativement peu fréquent dans le corpus.

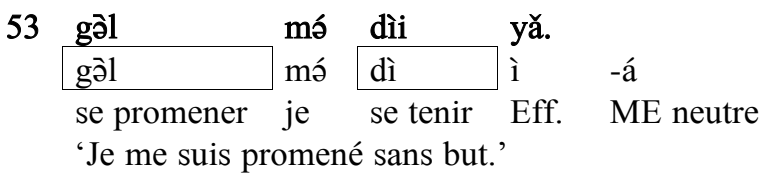

\section{Les verbes tantôt prospectifs, tantôt rétrospectifs}

- Dán 'équivaloir', 'suffire'

Le verbe dón est un verbe qui se construit régulièrement de façon intransitive et qui est susceptible de participer à plusieurs types de CV.

(i) Lorsqu'il est l'unique verbe d'un CV simple, ce verbe indique une égalité en taille ou le fait d'être suffisamment grand ou de proportion suffisante. La notion de taille est très présente dans le sémantisme de ce verbe. Il est d'ailleurs assurément dans un rapport de dérivation avec le verbe dá 'mesurer'.

En (54), dón notifie l'adéquation et signifie 'égaler, être de la même taille'.

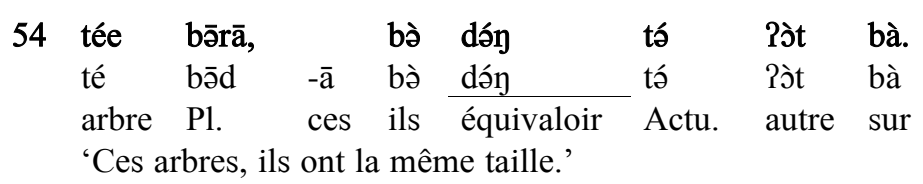

En (55), le verbe dán construit un CV simple dans lequel il véhicule l'idée de suffisance.

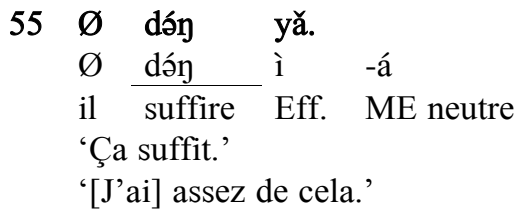


(ii) Lorsque dán est employé comme verbe prospectif dans un CV hiérarchisé, il exprime une modalité déontique de pouvoir. Cette modalité permissive est de l'ordre de l'autorisation liée à un statut social ou un âge, de l'interdiction ou de la proscription dans le cas d'une assertion négative (56).

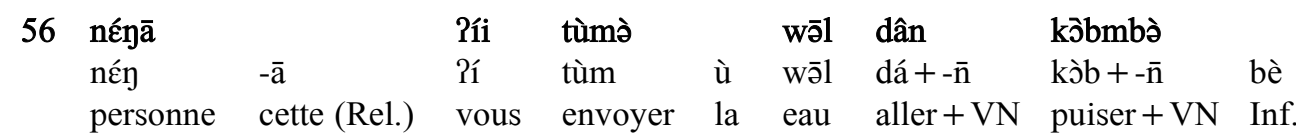

\begin{tabular}{|c|c|c|c|c|c|}
\hline \multirow[b]{2}{*}{$\varnothing$} & dán & wōl & nə̄ənàbà & & \\
\hline & dón & wāl & $n \bar{\partial}+-\bar{n}$ & -à & bè \\
\hline
\end{tabular}

la (Rel.) elle pouvoir eau boire +VN Dist. Inf. marigot dans

wōrò sé.

wādà sé?

là-bas Nég.

'La personne que vous envoyez chercher de l'eau n'a pas le droit de boire au marigot làbas.'

En (57), ce verbe indique à la fois l'inaptitude physique du varan à renverser l'hyène et l'incongruité d'un tel événement.

57 lígdō kân $\varnothing$ dán

varan vraiment il suffire Eff. log.sg.

làanbè.

là $+-\bar{n} \quad$ bè

renverser $+\mathrm{VN}$ Inf.

'[Hyène demande] comment Varan aurait pu la mettre à terre. ${ }^{27}$

En (58), on retrouve dans dán la notion de taille. Il s'agit là d'une modalité de pouvoir liée à une quantité.

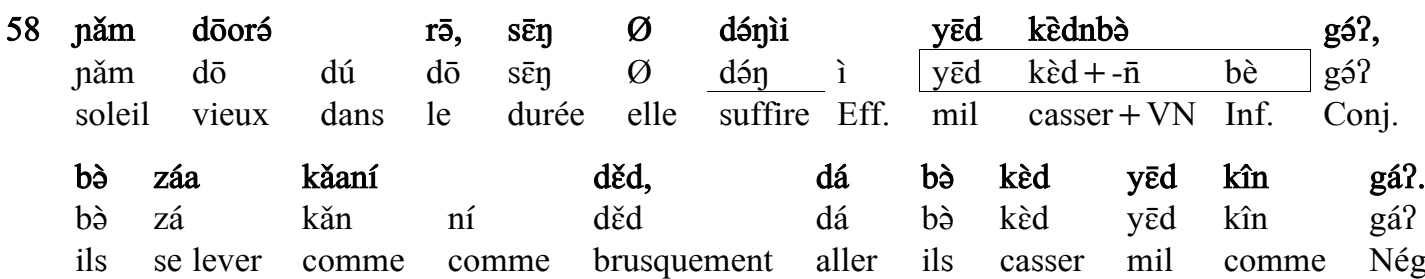

'Dans l'ancien temps, lorsque l'époque de la récolte du mil arrivait, on ne s'y mettait pas comme ça brusquement, on n'allait pas récolter le mil n'importe comment.'

(Littéralement '[...] quand la durée écoulée permet de/est suffisante pour casser le mil [...]')

(iii) Lorsque dón fonctionne comme verbe rétrospectif dans une série verbale, il exprime une modalité appréciative qui qualifie le procès de $\mathrm{V}_{1}$ de complet par rapport à une quantité attendue ou envisagée.

${ }^{27}$ Le connectif homophone de la marque de l'infinitif indique en (57) que le pronom tonique Pōbə̀n est le complément de l'infinitif. 
L'exemple (59) est un syntagme nominal constitué d'une proposition relative. Au sein de cette proposition, dón signale que les procès de $\mathrm{V}_{1}$ et de $\mathrm{V}_{2}$ ont atteint la quantité requise, que la part qui revient au sujet est suffisante.

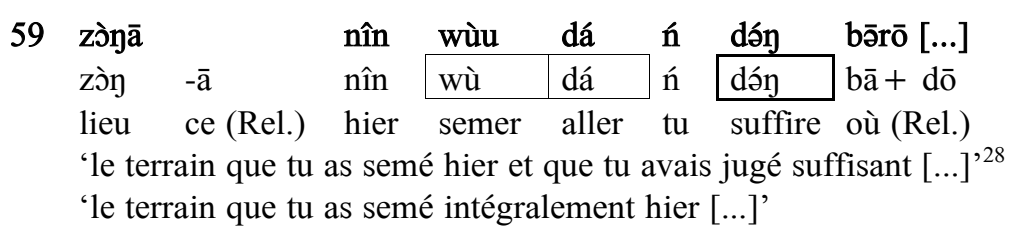

Si le sujet est pluriel, dán indique que les actants sont à égalité dans le domaine référentiel du procès du $V_{1}(60)$.

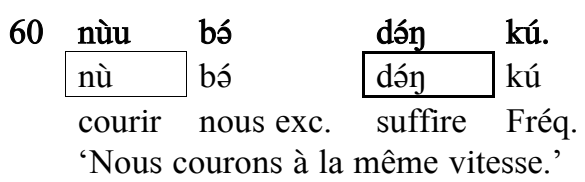

- Bán 'se rassembler', 'pouvoir', 'réussir'

Le verbe bán intervient dans trois types de CV : dans le CV simple, en tant que verbe prospectif et en tant que verbe rétrospectif dans des CV complexes.

(i) L'énoncé (61) illustre la construction simple de bán. Cette unique attestation peut suggérer que ce verbe exprime une comparaison aboutissant à une identité, mais d'autres attestations devront être recueillies pour valider cette hypothèse.
61

\begin{tabular}{lllll} 
wōl & $\varnothing$ & bán & yă. & \\
wōl & $\varnothing$ & bán & ì & -á \\
\cline { 5 - 6 } eau & elle & se rassembler & Eff. & ME neutre
\end{tabular}

'Les deux bras ne font plus qu'un.'

(Se dit à propos du confluent de deux cours d'eau.)

(ii) Lorsque bán fonctionne comme verbe prospectif dans un CV hiérarchisé, il véhicule une modalité déontique de l'ordre de la potentialité, de la capacité virtuelle à réaliser le procès du verbe régi $(62)^{29}$.

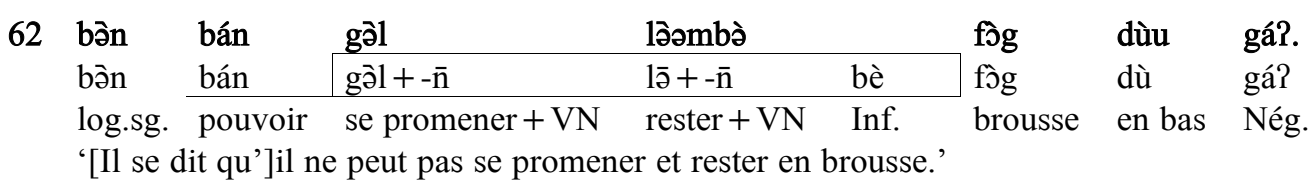

(iii) Dans la série verbale, bán peut s'employer comme verbe rétrospectif pour indiquer que les procès des premiers verbes de la série ont été menés à bien. Dans cette construction, bán véhicule une modalité appréciative et validante, la validation d'une capacité. Les énoncés (63) et (64) présentent cet emploi de bán où il signifie 'réussir, avoir pu mener à bien' le procès de $\mathrm{V}_{1}$.

${ }^{28}$ La position du verbe dá dans l'exemple (59) ne correspond pas à sa place habituelle (il intervient plutôt en début de série), mais les données ne nous permettent pas d'aller plus avant dans l'analyse.

${ }^{29}$ Dans le corpus, le verbe bán n'intervient que dans des énoncés négatifs ou interrogatifs. Il serait important de vérifier si son emploi dans un énoncé positif s'accorderait avec l'interprétation avancée ici. 
63 á?ā zàan mó bán gái.

Táiā zàn má bán

non nourrir ${ }^{30}$ je pouvoir Nég.

'Non, je ne réussirai pas l'élever.'

64 yāa kò?, nùu $\varnothing$ bán nōg gá?.

yā kò? nù $\varnothing$ bán nōg gá?

cheval aussi courir il pouvoir course Nég.

'En conséquence, le cheval ne réussit pas à courir.'

De nouvelles enquêtes devraient me permettre d'établir le lien sémantique entre le sens processif de bán et ses valeurs modales déontiques.

\section{S YNTHÈ SE}

Après avoir présenté le système verbal qui n'entre que peu en jeu dans l'expression de la modalité et de l'aspect, j'ai cherché, au travers de quelques exemples, à exposer le fonctionnement de la hiérarchisation et de la sérialisation verbale et à mettre en évidence le parallélisme qui réside entre la position du verbe principal et les valeurs structurelles de ces CV complexes, c'est-à-dire la correspondance entre la linéarité et le sémantisme de chaque structure, l'iconicité de ces constructions. Mon propos était de montrer comment, en exploitant à la fois les différents traits sémantiques de certains verbes et la variété des structures syntaxiques, cette langue supplée au développement relativement réduit de sa morphologie ${ }^{31}$.

L'étude de ces constructions a en effet montré que :

- par leur sémantisme, certains verbes ont une aptitude à fonctionner plus particulièrement comme verbe prospectif dans des CV hiérarchisés (ce sont ceux qui impliquent une visée) ou comme verbe rétrospectifs dans des séries verbales (ce sont ceux qui impliquent le terme du procès) ;

- le verbe conjugué tend à perdre sa valeur processive pour une valeur plus abstraite $^{32}$;

- cette valeur plus abstraite dépend du sémantisme du verbe et de la position respective de ce verbe et des autres bases verbales de la structure. En effet, placé en tête (CV hiérarchisé), le verbe principal implique une visée vers les procès des autres bases verbales (pouvoir + boire $\rightarrow$ pouvoir boire ; capacité virtuelle), alors que placé en fin de structure (série verbale), le procès du verbe principal est nécessairement postérieur à celui des autres

\footnotetext{
${ }^{30}$ Le premier sens du verbe zàn est 'nourrir, verser de la nourriture à des bêtes domestiques' ; par extension ce verbe prend le sens d'“élever un animal domestique'.

${ }^{31}$ D'autres domaines de la syntaxe de cette langue témoignent de procédés comparables quant à l'exploitation « intensive » du stock lexical et grammatical (détermination nominale, complétion verbale et discours rapporté notamment).

32 Dans le même registre, Durie (1997:323-324) signale aussi le cas des verbes 'donner' ou 'rencontrer' qui permettent d'introduire un argument supplémentaire (but ou bénéfactif) dans différentes langues.
} 
bases et peut donc le valider ou l'invalider (boire + pouvoir $\rightarrow$ réussir à boire ; capacité validée).

Le choix entre l'une et l'autre construction comprend donc, conjointement aux modalités (principalement déontiques et appréciatives) évoquées, une modalité épistémique : la construction à verbe prospectif implique que le locuteur considère le procès du verbe régi comme peu certain et/ou virtuel alors que la construction à verbe rétrospectif implique que le locuteur considère le procès du verbe précédent comme certain et réel au point de pouvoir apprécier son déroulement ou son résultat.

Les verbes qui s'éloignent de façon significative de leur sens processif dans les CV complexes soulèvent la question de leur possible grammaticalisation en tant qu'auxiliaires de conjugaison.

On a vu plus haut (paragraphe 2.1.3) que l'auxiliaire du futur dá peut s'analyser comme un cas de grammaticalisation du verbe dá 'aller' ; c'est d'ailleurs un cas attesté dans de nombreuses langues. Le fait est qu'une séquence comportant un CV hiérarchisé avec dá 'aller' pour verbe prospectif ne diffère d'un verbe régi par l'auxiliaire du futur que par la marque de l'infinitif ${ }^{33}$. Ceci pourrait plaider en faveur d'un accès privilégié de certains verbes prospectifs au rang d'auxiliaire de conjugaison.

D'autre part, les données montrent que certains verbes rétrospectifs présentent des spécificités que l'on peut interpréter comme les indices d'une grammaticalisation en cours.

En particulier, j'ai souligné

- $\quad$ sur le plan statistique, la situation particulière du verbe sà 'avoir l'habitude de' qui fonctionne très majoritairement comme verbe rétrospectif (une attestation originale et unique de son emploi en CV simple) et qui semble accepter une structure spécifique à deux indices sujet ;

- $\quad$ sur le plan syntaxique, le phénomène d'accord en transitivité, dans le cadre de la série verbale, pour deux couples de verbes d'une même classe dérivationnelle béd/bé 'finir' et bíd/bí 'rentrer'.

Aussi, étant donné la productivité et l'économie de ce système, la question de la grammaticalisation de ces verbes reste entière.

${ }^{33}$ Un CV hiérarchisé avec dá 'aller' peut évidemment apparaître au futur. 


\section{Abréviations utilisées}

\begin{tabular}{|c|c|c|c|}
\hline $\begin{array}{l}\text { Actu. } \\
\text { Aux. }\end{array}$ & $\begin{array}{l}\text { actualisateur } \\
\text { auxiliaire de conjugaison }\end{array}$ & $\begin{array}{l}\text { Interro. } \\
\text { IS }\end{array}$ & $\begin{array}{l}\text { marque d'interrogation } \\
\text { indice suiet }\end{array}$ \\
\hline $\mathrm{CN}$ & constituant nominal & $\log$ & logophorique \\
\hline Conj. & conjonction & $\mathrm{ME}$ & modalité d'énoncé \\
\hline Conn. & connectif & Nég. & particule négative \\
\hline $\mathrm{CV}$ & constituant verbal & $\mathrm{O}$ & objet \\
\hline Dist. & distanciatif & Obl. & obligatif \\
\hline Eff. & effectif & pl. & pluriel \\
\hline exc. & (nous) exclusif & Pl. & pluralisateur \\
\hline Fact. & dérivé factitif & Prog. & auxiliaire du progressif \\
\hline Fréq. & ME fréquentative & (Rel.) & frontières d'une proposition relative \\
\hline Fut. & auxiliaire du futur & $\mathrm{S}$ & sujet \\
\hline $\mathrm{H}$ & ton haut & sg. & singulier \\
\hline inc. & (nous) inclusif & V & verbe \\
\hline Inf. & infinitif & VN & verbonominal \\
\hline Int. & intensif & & \\
\hline
\end{tabular}

\section{Références}

Delplanque, A. (1998). Le mythe des « séries verbales ». Faits de langues, n¹1-12 Les langues d'Afrique subsaharienne, p. 231-250.

Durie, M. (1997). Grammatical Structures in Verb Serialization. In Complex Predicates (A. Alsina, J. Bresnan \& P. Sells eds), CSLI Lecture Notesn n64, Stanford, California.

Fabre, G. (2003). Le samba leko, langue Adamawa du Cameroun. München : Lincom Studies in African Linguistics 56.

Fardon, R. (1988). Between God, the Dead and the Wild, Chamba Interpretations of Ritual and Religion. London, Edinburgh University Press, for the International African Institute.

Greenberg, J. H., (1966 1 1 éd. 1963). The languages of Africa, Indiana UniversityBloomington, La Haye, Mouton.

Lord, C. (1993). Historical Change in Serial Verb Constructions. Amsterdam : John Benjamins.

Noye, D. (1989). Dictionnaire foulfouldé-français, Dialecte Peul du Diamaré NordCameroun. Garoua et Paris, Procure des Missions, Geuthner. 